\title{
Moderate and severe traumatic brain injury: effect of blood alcohol concentration on Glasgow Coma Scale score and relation to computed tomography findings
}

\author{
Nils Petter Rundhaug, MD, ${ }^{1}$ Kent Gøran Moen, MD, PhD, ${ }^{1,2}$ Toril Skandsen, MD, PhD, ${ }^{1,3}$ \\ Kari Schirmer-Mikalsen, MD, ${ }^{4,5}$ Stine B. Lund, MSc, ${ }^{2}$ Sozaburo Hara, MD, ${ }^{2}$ and Anne Vik, MD, PhD ${ }^{1,2}$ \\ Departments of ${ }^{1}$ Neuroscience and ${ }^{4}$ Circulation and Medical Imaging, Norwegian University of Science and Technology, \\ Trondheim; and Departments of ${ }^{2}$ Neurosurgery, ${ }^{3}$ Physical Medicine and Rehabilitation, and ${ }^{5}$ Anaesthesia and Acute Medicine, \\ St. Olavs Hospital, Trondheim University Hospital, Trondheim, Norway
}

OBJECT The influence of alcohol is assumed to reduce consciousness in patients with traumatic brain injury (TBI), but research findings are divergent. The aim of this investigation was to study the effects of different levels of blood alcohol concentration (BAC) on the Glasgow Coma Scale (GCS) scores in patients with moderate and severe TBI and to relate the findings to brain injury severity based on the admission CT scan.

METHODS In this cohort study, 265 patients (age range 16-70 years) who were admitted to St. Olavs University Hospital with moderate and severe TBI during a 7-year period were prospectively registered. Of these, 217 patients (82\%) had measured BAC. Effects of 4 BAC groups on GCS score were examined with ordinal logistic regression analyses, and the GCS scores were inverted to give an OR $>1$. The Rotterdam CT score based on admission CT scan was used to adjust for brain injury severity (best score 1 and worst score 6 ) by stratifying patients into 2 brain injury severity groups (Rotterdam CT scores of 1-3 and 4-6).

RESULTS Of all patients with measured BAC, $91 \%$ had intracranial CT findings and $43 \%$ had BAC > $0 \mathrm{mg} / \mathrm{dl}$. The median GCS score was lower in the alcohol-positive patients (6.5, interquartile range $[I Q R] 4-10)$ than in the alcohol-negative patients $(9$, IQR $6-13 ; p<0.01)$. No significant differences were found between alcohol-positive and alcohol-negative patients regarding other injury severity variables. Increasing BAC was a significant predictor of lower GCS score in a dose-dependent manner in age-adjusted analyses, with OR 2.7 (range 1.4-5.0) and 3.2 (range 1.5-6.9) for the 2 highest BAC groups $(p<0.01)$. Subgroup analyses showed an increasing effect of BAC group on GCS scores in patients with Rotterdam CT scores of 1-3: OR 3.1 (range 1.4-6.6) and 6.7 (range 2.7-16.7) for the 2 highest BAC groups $(p<0.01)$. No such relationship was found in patients with Rotterdam CT scores of 4-6 ( $p=0.14-0.75)$.

CONCLUSIONS Influence of alcohol significantly reduced the GCS score in a dose-dependent manner in patients with moderate and severe TBI and with Rotterdam CT scores of 1-3. In patients with Rotterdam CT scores of 4-6, and therefore more CT findings indicating increased intracranial pressure, the brain injury itself seemed to overrun the depressing effect of the alcohol on the CNS. This finding is in agreement with the assumption of many clinicians in the emergency situation.

http://thejns.org/doi/abs/10.3171/2014.9.JNS14322

KEY WORDS ethanol; craniocerebral trauma; x-ray computed tomography; intracranial hypertension; consciousness disorders; traumatic brain injury

$\mathrm{T}$ RAUMATIC brain injury (TBI) is a major cause of morbidity and mortality in both low- and high-income countries. ${ }^{12}$ Severity classification of TBI is mostly based on the Glasgow Coma Scale (GCS) score, which is the most-used clinical tool to assess patients with reduced consciousness. ${ }^{3,19,24}$ Hence, obtaining valid GCS scores is important to give the patient adequate care at the right treatment level. $3,8,25$

ABBREVIATIONS AIS = Abbreviated Injury Scale; BAC = blood alcohol concentration; EDH = epidural hemorrhage; GCS = Glasgow Coma Scale; GOSE = Glasgow Outcome Scale Extended; ICP = intracranial pressure; IQR = interquartile range; ISS = Injury Severity Score; TAI = traumatic axonal injury; $T B I=$ traumatic brain injury. SUBMITTED February 16, 2014. ACCEPTED September 3, 2014.

INCLUDE WHEN CITING Published online October 31, 2014; DOI: 10.3171/2014.9.JNS14322.

DISCLOSURE During part of the study period, Dr. Schirmer-Mikalsen received a research grant from the Liaison Committee between the Central Norway Regional Health Authority and the Norwegian University of Science and Technology. The authors report no conflict of interest concerning the materials or methods used in this study or the findings specified in this paper. 
Alcohol intoxication is frequent among adult patients with TBI admitted to emergency departments, ${ }^{10,23}$ although large studies in which a high percentage of patients with measured blood alcohol concentrations (BACs) are included are sparse. Among health care professionals it is commonly assumed that alcohol reduces consciousness in patients with TBI, thereby limiting the clinical applicability of the GCS score in intoxicated patients. If a low GCS score in alcohol-positive patients is incorrectly attributed to alcohol, the result could be an underestimation of injury severity and unnecessary delay of important diagnostic and therapeutic interventions. ${ }^{21}$ On the other hand, overestimation of injury severity could result in overconsumption of expensive, highly specialized treatment, including unnecessary and potentially risky intervention.

Previous studies have failed to show a consistent relationship between BAC and level of consciousness in patients with TBI. Some studies have found that high BAC lowers GCS scores, $, 1,4,6,13$ whereas 2 large-scale studies concluded that no clinically significant effect existed. ${ }^{17,21}$ In the latter studies brain injury severity was controlled for by using the Abbreviated Injury Scale (Head AIS). In a more recent study, Lange et al. studied the effect of alcohol in patients with (23.4\%) and without intracranial traumatic findings based on CT scans from the day of injury. ${ }^{7}$ Most patients had mild TBI, and an effect of alcohol on GCS score was found in only a small subgroup of patients $(n=14)$ with traumatic intracranial CT findings, lower GCS scores, and a high BAC. In contrast to patients with mild TBI, most patients with moderate and severe TBI will have abnormal findings on CT scans obtained at admission. Hence, it is possible to further adjust for brain injury severity with methods that incorporate CT findings indicating increased intracranial pressure (ICP) that affects consciousness. To our knowledge, no such study has previously been performed.

The aim of this study was to assess the effect of alcohol on GCS score in patients with moderate and severe TBI, and also to adjust for brain injury severity based on admission Rotterdam CT score. ${ }^{9}$ We used 4 BAC groups and hypothesized that higher BAC would predict lower GCS scores. We divided the patients into 2 groups based on the Rotterdam CT score to study if the influence of alcohol would have a greater effect in patients with fewer CT findings and thus also fewer signs of increased ICP.

\section{Methods \\ Patient Population}

All patients with moderate-to-severe head injuries according to the Head Injury Severity Scale (or HISS) ${ }^{18}$ who were admitted to St. Olavs University Hospital in Trondheim, Norway, during a 7-year period (October 2004-October 2011) were registered $(n=399)$. The hospital is the only Level I trauma center in a region of 680,000 inhabitants. Of all registered patients, 265 were between 16 and 70 years old, and of these, 48 patients had missing BAC values, leaving 217 patients (82\%) for further analysis. Although the legal drinking age in Norway is 18 years, we decided to include patients between 16 and 18 years because underage drinking is common. Injury severity and outcome data on the patients with missing BAC values are also presented, to evaluate if there were any selection bias. Intoxicated patients, in whom mild TBI had been evaluated because of falsely low GCS scores during the first hours postinjury, were not included..$^{1,20}$

\section{Injury Variables and Outcome Assessment}

All prehospital and in-hospital variables were collected prospectively during the study period. The GCS scores were collected at the accident scene (by anesthesiologists), the primary hospital (by residents in general surgery), or on admission to the Level I trauma center (by residents in neurosurgery). Preintubation GCS scores were used for intubated patients, whereas the GCS scores obtained on admission were used for the nonintubated patients $(\mathrm{n}=95)$. In 2 patients the GCS scores before intubation at the primary hospital were missing, and prehospital scores were used.

Secondary events defined as prehospital or admission hypoxia $\left(\mathrm{O}_{2}\right.$ saturation $\left.<92 \%\right)$ and/or hypotension (systolic blood pressure $<90 \mathrm{~mm} \mathrm{Hg}$ ), any pupillary dilation (prehospital and/or on admission), and intubation status were registered. Injury Severity Scores (ISSs) were estimated for all patients (by S.H. and K.G.M.) and used as a measure for overall injury severity. ${ }^{2}$ Global outcome was assessed 12 months postinjury by telephone or personal contact, using the structured interview for the Glasgow Outcome Scale Extended (GOSE). We used the 6-month outcome if the 12-month GOSE score was missing ( $\mathrm{n}=$ 7). In addition, 1 patient died of a cause other than the TBI, 1 patient could not be assessed, and 9 patients were lost to follow-up in the whole cohort.

\section{Classification of CT Scans}

The CT scans obtained on admission were reviewed by a radiologist (I.H.S.; see Acknowledgments) or a resident in neurosurgery (K.G.M.) in cooperation with 3 neuroradiologists and classified according to the Rotterdam CT classification (best score 1, worst score 6). ${ }^{9}$ This classification is based on CT findings found to have prognostic value in TBI, such as midline shift and compression of basal cisterns (signs of increased ICP), traumatic subarachnoid hemorrhage or intraventricular hemorrhage, and the presence of epidural hemorrhage ([EDH] reducing the score by 1). The Rotterdam CT score was used to adjust for injury severity by dichotomization, yielding 2 groups based on the severity of the radiological findings; Rotterdam CT scores of 1-3 and Rotterdam CT scores of 4-6. To eliminate the effect of subtracting 1 point from patients with $\mathrm{EDH}$, we also categorized without subtracting 1 point and evaluated the effect on the overall results again. Seven patients then changed category; from Rotterdam CT scores of $1-3$ to $4-6$.

\section{Determination of BAC}

Blood samples for alcohol screening are routinely collected from all patients older than 16 years on admission at St. Olavs University Hospital with moderate and severe TBI. The BAC value is expressed in milligrams per deciliter. We used an enzymatic method for determining BAC 
from plasma based on the reaction of alcohol dehydrogenase on alcohol and conversion of nicotinamide adenine dinucleotide (NAD) to NAD + hydrogen (NADH). The alcohol concentration is indirectly determined through a constant relationship with the NADH concentration, measured with spectrophotometry at $340 \mathrm{~nm}$. In our study an approved system by Roche Modular P was used (NS-EN ISO/IEC 17025).

The patients were described as alcohol positive (BAC $>0 \mathrm{mg} / \mathrm{dl}$ ) and alcohol negative (BAC $0 \mathrm{mg} / \mathrm{dl})$. The patients were also divided into 4 BAC groups according to recommendations given by Lange et al. ${ }^{7}$ The groups were slightly different from those used in that study, to secure more even numbers: BAC $0 ;>0$ to $<150 ; 150$ to $<250$; and $\geq 250 \mathrm{mg} / \mathrm{dl}$. It should therefore be noted that this grouping was not based on a clinical BAC scale.

\section{Statistical Analysis}

Patient and injury characteristics are given as percentages, mean $\pm \mathrm{SD}$, and median with interquartile range (IQR). Distribution of data was tested using quantilequantile plots and the Shapiro-Wilk test for normal distribution. The GCS score was not normally distributed, and nonparametric tests (Mann-Whitney U-test) were used for analyses. For categorical variables the chi-square or the Fisher exact test was used.

Scatterplots were first visually inspected to find relationships between BAC and age, GCS score, ISS, and Rotterdam CT score. The Spearman correlation coefficient was then used to quantify any correlations between the variables. A linear nonparametric trend test (Stata command: nptrend) was used to test for trends in GCS score with increasing Rotterdam CT score. Ordinal logistic regression with GCS score as the dependent variable and age and BAC as explanatory variables was used to test the effects of BAC. Odds ratios were calculated for each BAC group, with the BAC group $=0$ as the reference. The GCS scores were inverted to give an OR $>1$ and were thus more intuitive to interpret. To evaluate whether the effect of alcohol depended on brain injury severity, analyses were performed separately in the 2 subgroups of patients based on the Rotterdam CT score (scores of 1-3 and 4-6).

The statistical significance level was set to $\mathrm{p}<0.05$ (2-sided) and a 95\% confidence interval was used. The data were analyzed using IBM SPSS Statistics version 21.0 and STATA version 12.0 for Mac.

\section{Ethics Approval}

The Regional Committee for Medical Research Ethics approved the study. Written informed consent was obtained from surviving patients or, for incapacitated individuals, their next of kin. The Norwegian Directorate of Health approved use of data from deceased individuals.

\section{Results}

\section{Demographic and Injury Variables in the TBI Cohort}

There were no significant differences between patients included in the analysis and those excluded due to missing $\mathrm{BAC}$ values, but the latter tended to be older and to have higher GCS scores (Table 1).
Table 2 shows demographic and clinical data for the 217 patients with measured BAC. Of these, 94 patients (43\%) were alcohol positive. The median GCS score of all patients was 8 (IQR 5-12), and 91\% of the patients had intracranial CT findings. A total of $72 \%$ had Rotterdam CT scores of 1-3, and 28\% had Rotterdam CT scores of 4-6; also, 43\% were alcohol positive in both groups.

\section{Comparison of Alcohol-Positive and Alcohol-Negative Patients}

The median GCS score was significantly lower in the alcohol-positive patients $(6.5$, IQR $4-10)$ than in the alcohol-negative patients (9, IQR 6-13; p < 0.01) (Table 2). More alcohol-positive patients $(65 \%)$ had GCS scores $\leq 8$ than alcohol-negative patients $(46 \%, \mathrm{p}<0.01)$. The median GCS scores by BAC group are presented in Fig. 1. A significant linear decrease in GCS score with increasing Rotterdam CT score was observed among alcohol-negative patients $(\mathrm{p}<0.001)$ (Table 3$)$.

Falls were the most common cause of injury among alcohol-positive patients (49\%), whereas motor vehicle accidents was the most common cause in the alcoholnegative group $(56 \%)(\mathrm{p}<0.01)$. A larger proportion of alcohol-negative patients were intubated at the scene of the accident $(p=0.045)$. There were no significant differences in age, pupillary dilation, presence of prehospital and/or admission hypoxia and hypotension, ISS, Rotterdam CT scores, or GOSE scores between alcohol-positive and alcohol-negative patients (Table 2).

\section{Prediction of GCS Score by BAC Group}

In the whole patient group, BAC significantly predicted lower GCS score in a dose-dependent manner in age-adjusted analyses (Table 4; Fig. 1). When the patients were stratified according to injury severity, we found that BAC significantly predicted lower GCS score only among patients with Rotterdam CT scores of 1-3. Again, the decline in GCS score was observed with increasing $\mathrm{BAC}$ group and the prediction was significant for $\mathrm{BAC} \geq$ $150 \mathrm{mg} / \mathrm{dl}$. In contrast, BAC was not a predictor for GCS score in patients with Rotterdam CT scores of 4-6. The BAC and GCS scores correlated significantly $(r=-0.245$, $\mathrm{p}<0.01$ ), but otherwise no significant correlation between BAC, age, and other injury variables (ISS or Rotterdam CT score) was found.

The reclassification of 7 patients with EDH from Rotterdam CT scores of 1-3 to 4-6 did not change the results substantially (data not shown).

\section{Discussion}

The main finding in this prospective study of patients with moderate and severe TBI was that alcohol reduced GCS scores. We found that GCS scores gradually decreased when BAC group increased. Interestingly, subgroup analysis showed that this relationship was only found among patients with a Rotterdam CT score of 1-3, and not among patients with higher Rotterdam scores and consequently more CT findings and signs of increased ICP. 
TABLE 1. Characteristics, injury-related variables, and outcome in the total cohort of 265 patients with TBI

\begin{tabular}{lccc}
\hline \multicolumn{1}{c}{ Variable } & BAC Measured & BAC Not Measured & p Value \\
\hline No. of patients & 217 & 48 & 0.070 \\
\hline Median age in yrs (IQR) & $38.0(22.9-52.4)$ & $46.2(24.3-60.8)$ & 0.249 \\
\hline M/F sex (\%) & $172 / 45(79 / 21)$ & $34 / 14(71 / 29)$ & 0.483 \\
\hline Injury mechanism (\%) & & & \\
\hline Vehicle accident & $105(49)$ & $20(42)$ & \\
\hline Fall & $81(37)$ & $23(48)$ & 0.508 \\
\hline Other & $26(12)$ & $3(6)$ & 0.074 \\
\hline Missing & $5(2)$ & $2(4)$ & 0.506 \\
\hline Intubated at scene of accident (\%) & $81(37)$ & $15(31)$ & 0.526 \\
\hline Median GCS score (IQR) & $8(5-12)$ & $10(6-13)$ & 0.678 \\
\hline Median ISS score (IQR) & $25(17-32)$ & $3(2-4)$ & $6(4-8)$ \\
\hline Median Rotterdam CT score (IQR) & $3(2-4)$ & $6(5-8)$ & \\
\hline Median GOSE score (IQR)
\end{tabular}

* Total for this variable was 254; 9 were lost to follow-up, 1 died of other causes, and 1 could not be assessed.

\section{Influence of Alcohol and Effect on GCS Score}

Our results demonstrating a decrease of the GCS score with higher BAC levels in patients with TBI are in accordance with some previous studies. . $^{1,4,6,13}$ In the most recent study, Shahin et al. found larger differences between GCS score on admission and best Day 1 GCS score in intoxicated patients $(\mathrm{BAC}>80 \mathrm{mg} / \mathrm{dl})$ than in the nonintoxicated patients. ${ }^{13}$ In contrast to this, 2 other studies failed to demonstrate a clinically significant effect of alcohol in the vast majority of TBI patients. ${ }^{7,17}$ However, subgroup analyses in these 2 studies showed some effect in the more injured patients. Sperry et al. found a clinically significant effect (defined as $>1$ point GCS score difference) of alcohol among intoxicated patients (BAC $>80 \mathrm{mg} / \mathrm{dl}$ ) in the most injured patients: those with head AIS scores of $5 .{ }^{17}$ A limitation of their study is that BAC was measured selectively, and they do not report the percentage of patients from the total cohort who had measurement of BAC.

Also, in the study by Lange et al., in which $62 \%$ of all patients had a GCS score of 15 , no effect was found in most TBI patients. However, in a small subgroup (n $=14)$ of patients with high BAC $(>200 \mathrm{mg} / \mathrm{dl})$ and intracranial abnormalities detected on CT scanning, GCS scores were reduced. ${ }^{7}$ On the other hand, Stuke et al. ${ }^{21}$ showed no clinically significant effect even in subgroups of patients with head AIS scores of 3-6 in their comprehensive registry study. They included the whole severity spectrum of patients with and without TBI, in which only $45 \%$ of the registered patients had BAC measured. Also, the exact BAC values were not available, and therefore difference in GCS scores were only studied in alcoholpositive (BAC-positive) versus alcohol-negative patients.

\section{Subgroup Analyses of Patients With Different Brain Injury Severity}

We observed that the effect on GCS score was limited to patients with Rotterdam CT scores of 1-3. These patients typically have cortical contusions and/or hemorrhages, mostly with no signs of increased ICP, and $9 \%$ of the patients in our study even had no CT findings. Patients with Rotterdam CT scores of 4-6 all have signs of increased ICP (midline shift and/or compressed or absent basal cisterns), which reduces consciousness in patients with acute TBI. ${ }^{5}$ Thus, it is likely that the CNS-depressing effect from alcohol is overrun by the brain injury itself in those patients. This finding is in agreement with the assumptions of many clinicians that alcohol is not important for level of consciousness in the more severely injured patients.

To study alcohol effects on GCS score, one should adjust for other factors known to affect consciousness, such as increased ICP and traumatic axonal injury (TAI). ${ }^{15}$ Therefore, we used the Rotterdam CT score calculated from visual inspection of the admission CT scans to stratify patients into 2 brain injury severity groups. This was done because findings that are associated with increasing ICP were presumed to correlate with increasing CT score for most patients. ${ }^{11}$ We found a significant linear decrease in GCS score with increasing Rotterdam CT score in alcohol-negative patients, a finding that also supported our use of this method. In addition, the occurrence of intraventricular and/or subarachnoid hemorrhage often found in patients with TAI will add 1 point to the score. ${ }^{16}$

\section{Effect of Alcohol in Patients With Moderate and Severe TBI}

In comparison with most other studies, we included only patients with moderate and severe TBI (the median GCS score was 8), and almost all of our patients had intracranial CT findings. All patients included had some degree of alteration in mental status or focal neurological deficits, and it therefore seems that BAC can be a source of error in the early neurological assessment of patients with moderate and severe TBI. We could speculate that patients with moderate and severe TBI may experience a synergistic effect between alcohol and the brain injury during the first hours postinjury? 
TABLE 2. Characteristics, injury-related variables, and outcome in alcohol-negative versus alcohol-positive patients with TBI

\begin{tabular}{|c|c|c|c|c|}
\hline Variable & All Patients & Alcohol Negative & Alcohol Positive & $p$ Value \\
\hline No. of patients & 217 & 123 & 94 & \\
\hline Median age in yrs (IQR) & $35.6(22.9-52.4)$ & $39.4(23.7-53.3)$ & $28.2(21.8-50.5)$ & 0.247 \\
\hline$M / F \operatorname{sex}(\%)$ & $172 / 45(79 / 21)$ & $91 / 32(74 / 26)$ & $81 / 13(86 / 14)$ & $0.02^{*}$ \\
\hline \multicolumn{5}{|l|}{ Injury mechanism (\%) } \\
\hline Vehicle accident & $105(49)$ & $69(56)$ & $36(38)$ & $0.009^{*}$ \\
\hline Fall & $81(37)$ & $35(29)$ & $46(49)$ & $0.002^{*}$ \\
\hline Other & $26(12)$ & $16(13)$ & $10(11)$ & 0.594 \\
\hline Unknown & $5(2)$ & $3(2)$ & $2(2)$ & \\
\hline Intubated at scene of accident (\%) & $81(37)$ & $53(43)$ & $28(30)$ & $0.045^{*}$ \\
\hline Median GCS score (IQR) & $8(5-12)$ & $9(6-13)$ & $6.5(4-10)$ & $0.001^{*}$ \\
\hline $3-8(\%)$ & $118(54)$ & $57(46)$ & $61(65)$ & $0.007^{*}$ \\
\hline $9-13$ & $85(39)$ & $57(46)$ & $28(30)$ & $0.013^{*}$ \\
\hline $14-15 \dagger$ & $14(7)$ & $9(8)$ & $5(5)$ & 0.552 \\
\hline \multicolumn{5}{|l|}{ Pupillary dilation (\%) } \\
\hline Normal & $182(84)$ & $100(81)$ & $82(87)$ & 0.239 \\
\hline Unilat & $24(11)$ & $14(11)$ & $10(11)$ & \\
\hline Bilat & $10(5)$ & $8(7)$ & $2(2)$ & \\
\hline Missing & $1(<1)$ & $1(<1)$ & $0(0)$ & \\
\hline Median BAC in mg/dl (IQR) & & & $187(137-250)$ & \\
\hline $0(\%)$ & & $123(100)$ & 0 & \\
\hline$>0$ to $<150$ & & & $27(29)$ & \\
\hline 150 to $<250$ & & & $44(47)$ & \\
\hline$\geq 250$ & & & $23(24)$ & \\
\hline Median ISS (IQR) & $25(17-31.5)$ & $25(18-33)$ & $25(16-30)$ & 0.146 \\
\hline \multicolumn{5}{|l|}{ Secondary events $\ddagger$ (\%) } \\
\hline Hypoxia; $\mathrm{SaO}_{2}<92 \%$ & $39(18)$ & $23(19)$ & $16(17)$ & 0.498 \\
\hline Systolic BP <90 mm Hg & $19(9)$ & $10(8)$ & $9(10)$ & 0.810 \\
\hline Missing & $10(5)$ & $7(6)$ & $3(3)$ & \\
\hline Median Rotterdam CT score (IQR) & $3(2-4)$ & $3(2-4)$ & $3(2-4)$ & 0.574 \\
\hline $1-3(\%)$ & $157(72)$ & $89(72)$ & $68(72)$ & 0.998 \\
\hline $4-6$ & $60(28)$ & $34(28)$ & $26(28)$ & 0.998 \\
\hline Median GOSE score (IQR)§ & $6(5-8)$ & $6(5-8)$ & $7(5-8)$ & 0.450 \\
\hline
\end{tabular}

\section{Alcohol Dose Dependency}

We used 4 BAC groups to study the effect of alcohol on the GCS score, and therefore we were able to test for dose-response effects. The effect of increasing BAC was dose dependent, so that patients in the higher BAC groups $(\geq 150 \mathrm{mg} / \mathrm{dl})$ had significantly lower GCS scores than those in the alcohol-negative group. This is in accordance with some of the findings in the study of Lange et al., in which high BAC (>200 mg/dl) was found to reduce GCS scores in 14 patients with abnormal CT scans. ${ }^{7}$ Several other studies have, however, used a single cutoff for BAC to classify patients into 2 groups. ${ }^{13,17,21}$ Thus, they fail to identify any dose-dependent relationships between BAC and GCS scores, and the effects from very high BAC may be missed. Large individual differences in alcohol tolerance also militate against the use of a single cutoff value. ${ }^{22}$ It should be noted, however, that the BAC grouping was not based on a clinical scale but rather was chosen to get more even groups.

\section{Incidence of Alcohol-Positive Patients in the TBI Cohort}

In our cohort of patients with moderate and severe TBI, $43 \%$ had BAC $>0$. However, because $18 \%$ of all patients had no BAC measurement, the exact percentage may be slightly different. Although the patients without BAC were similar to the patients with BAC with regard to most injury variables and outcomes, there was a tendency to older age and higher GCS scores in the former group. 

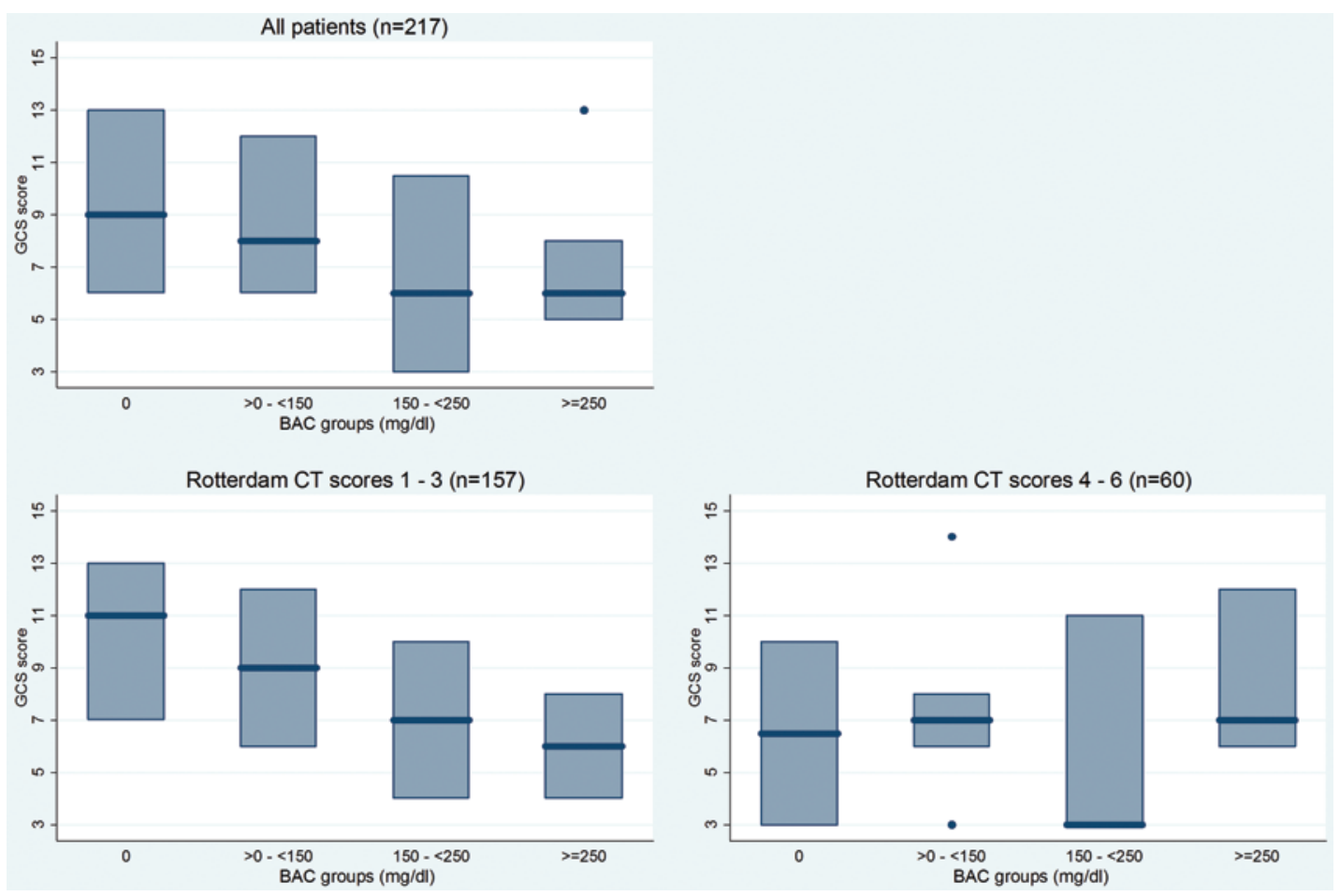

FIG. 1. Box plots showing change in GCS score with increasing BAC group; the median GCS score and IQR in the 4 BAC groups for all TBI patients and by brain injury severity group are shown. Upper Left: All TBI patients. Lower Left: Patients with Rotterdam CT scores of 1-3. Lower Right: Patients with Rotterdam CT scores of 4-6. Circles denote outliers; each represents 1 patient. Figure is available in color online only.

Thus we would suggest that fewer patients were alcohol positive among patients with missing BAC, and $43 \%$ is probably a marginal overestimation.

\section{Strengths and Limitations of the Study}

The sample size of 217 patients was large in this prospective TBI study. The fact that $82 \%$ of the patients in the total cohort had their BAC measured is a strength; most alcohol TBI studies have a much lower percentage ${ }^{7,13,21}$ or have not reported it. ${ }^{17}$

Brain injury severity was measured with the Rotterdam CT score, which is an injury scoring system based on admission CT scans that includes factors known to affect consciousness in patients with TBI. However, a limitation of the Rotterdam CT classification is that an epidural mass lesion reduces the score by 1 , since this scale was developed as a prognostic measure. Therefore we also performed the analyses by reclassifying the $7 \mathrm{pa}-$ tients with EDH into the group with Rotterdam CT scores of 4-6. The results were the same. Also, some of these patients with severe TAI and no intracranial hypertension were still classified in the brain injury severity group with Rotterdam CT scores of 1-3. Thus, it would have been preferable to also adjust for TAI lesions on the MRI study, and especially the occurrence of, for example, bilateral TAI brainstem lesions. ${ }^{14}$ However, because MRI is currently not a part of the routine acute examination, ${ }^{14}$ this neuroimaging modality was performed in only a limited

TABLE 3. Comparison of GCS score in the 6 different Rotterdam CT categories in alcohol-negative and alcohol-positive patients

\begin{tabular}{cccccc}
\hline $\begin{array}{c}\text { Rotterdam } \\
\text { CT Score }\end{array}$ & $\begin{array}{c}\text { No. Alcohol } \\
\text { Negative (\%) }\end{array}$ & $\begin{array}{c}\text { Median GCS Score (IQR) in } \\
\text { Alcohol-Negative Group* }\end{array}$ & $\begin{array}{c}\text { No. Alcohol } \\
\text { Positive (\%) }\end{array}$ & $\begin{array}{c}\text { Median GCS Score (IQR) in } \\
\text { Alcohol-Positive Group† }\end{array}$ & $\begin{array}{c}\text { Median BAC (IQR) in } \\
\text { Alcohol-Positive Group }\end{array}$ \\
\hline 1 & $3(2)$ & $7(6-15)$ & $4(4)$ & $7(5-9)$ & $171.0(69.6-231.9)$ \\
\hline 2 & $42(34)$ & $11(7-13)$ & $24(26)$ & $6.5(3-9.0)$ & $184.8(127.2-225.3)$ \\
\hline 3 & $44(36)$ & $9.5(7-13)$ & $40(43)$ & $8(5-11)$ & $199.1(137.7-276.1)$ \\
\hline 4 & $19(15)$ & $7.0(3-11)$ & $14(15)$ & $7.5(3-12)$ & $184.6(134.7-255.8)$ \\
\hline 5 & $10(9)$ & $6(4.75-7)$ & $8(9)$ & $4.5(3-7)$ & $195.6(166.0-258.1)$ \\
\hline 6 & $5(4)$ & $3(3-5)$ & $4(4)$ & $4.5(3-7)$ & $174.4(117.4-186.2)$ \\
\hline Total & 123 & & 94 & & \\
\hline
\end{tabular}

${ }^{*}$ A decreasing trend in the median GCS score was observed among alcohol-negative patients $(p<0.001)$.

$\dagger$ No trend in median GCS score was observed among alcohol-positive patients $(p=0.266)$. 
TABLE 4. Ordinal regression analysis: GCS score predicted by BAC group, adjusted for age*

\begin{tabular}{cclc}
\hline \multicolumn{1}{c}{ BAC Group } & No. $(\%)$ & OR $(95 \% \mathrm{Cl})$ & p Value \\
\hline All patients & 217 & & \\
\hline $0 \mathrm{mg} / \mathrm{dl}$ & $123(57)$ & 1.0 & Reference \\
\hline$>0$ to $<150 \mathrm{mg} / \mathrm{dl}$ & $27(12)$ & $1.0(0.5-2.1)$ & 0.929 \\
\hline 150 to $<250 \mathrm{mg} / \mathrm{dl}$ & $44(20)$ & $2.7(1.4-5.0)$ & 0.003 \\
\hline$\geq 250 \mathrm{mg} / \mathrm{dl}$ & $23(11)$ & $3.2(1.5-6.9)$ & 0.003 \\
\hline Rotterdam CT Score 1-3 & 157 & & \\
\hline $0 \mathrm{mg} / \mathrm{dl}$ & $89(57)$ & 1.0 & Reference \\
\hline$>0$ to $<150 \mathrm{mg} / \mathrm{dl}$ & $21(13)$ & $1.5(0.6-3.4)$ & 0.383 \\
\hline 150 to $<250 \mathrm{mg} / \mathrm{dl}$ & $29(18)$ & $3.1(1.4-6.6)$ & 0.004 \\
\hline$\geq 250 \mathrm{mg} / \mathrm{dl}$ & $18(11)$ & $6.7(2.7-16.7)$ & $<0.001$ \\
\hline Rotterdam CT Score 4-6 & 60 & & \\
\hline $0 \mathrm{mg} / \mathrm{dl}$ & $34(57)$ & 1.0 & Reference \\
\hline$>0$ to $<150 \mathrm{mg} / \mathrm{dl}$ & $6(10)$ & $0.4(0.1-1.9)$ & 0.252 \\
\hline 150 to $<250 \mathrm{mg} / \mathrm{dl}$ & $15(25)$ & $2.5(0.7-8.5)$ & 0.137 \\
\hline$\geq 250 \mathrm{mg} / \mathrm{dl}$ & $5(8)$ & $0.8(0.2-3.8)$ & 0.752 \\
\hline$*$
\end{tabular}

* GCS scores are inverted to give an OR > 1 and are thus more intuitive to interpret.

number of the cohort, and further adjustment for lesions known to be a sign of severe TAI could not be done in this study. Moreover, there is no reason to believe that TAI should be more prevalent among the alcohol-positive patients, because traffic accidents were more common causes of injury in the alcohol-negative group.

The sample size in BAC subgroups with Rotterdam CT scores of 4-6 is small, and a Type II error cannot be ruled out. We did not adjust for variations in time from injury to GCS score evaluation and blood sampling. It is also likely that another grouping of BAC values would have given a significant decline in GCS score at a different BAC level than we observed. ${ }^{7}$ Possible effects from drug use and abuse of illegal substances are not documented in this patient material and make up a possible confounding factor.

\section{Conclusions}

In this prospective study we found that the influence of alcohol may be a source of error in the assessment of reduced consciousness in patients with moderate and severe TBI. The reduction of GCS score with increasing BAC was only found in patients with Rotterdam CT scores of $1-3$. Such patients typically have cortical contusions and/ or hemorrhages, mostly without any signs of increased ICP, and some even have no CT findings. No such relationship was found among patients with more CT findings and signs of increased ICP (Rotterdam CT scores of 4-6). This finding is in agreement with the assumption of many clinicians that alcohol is not of importance for level of consciousness in the most severely injured patients. Hence, there is a risk of overestimating the injury severity among some alcohol-positive patients without severe CT findings who are assumed to have a moderate or even severe TBI during neurological assessment in the emergency phase. A liberal wake-up regimen in the intensive care unit setting in some patients might be supported based on our findings. Reduced GCS scores must not be attributed, however, to alcohol intoxication before a head CT scan is performed, and also, some patients may have severe TAI that could explain a low GCS score despite no or modest injury on CT scan.

\section{Acknowledgments}

We thank Stine Borgen Lund, Otto Aarhaug, and Beate Holmqvist Karlsen for the management of the patient database and performing the GOSE interviews and Ingrid Haavde Strand (I.H.S.) for calculating the Rotterdam CT scores.

\section{References}

1. Alexander S, Kerr ME, Yonas H, Marion DW: The effects of admission alcohol level on cerebral blood flow and outcomes after severe traumatic brain injury. J Neurotrauma 21:575583,2004

2. Baker SP, O'Neill B, Haddon W Jr, Long WB: The injury severity score: a method for describing patients with multiple injuries and evaluating emergency care. J Trauma 14:187196,1974

3. Brain Trauma Foundation, American Association of Neurological Surgeons, Congress of Neurological Surgeons, AANS/CNS Joint Section on Neurotrauma and Critical Care: Guidelines for the management of severe traumatic brain injury, 3rd edition. J Neurotrauma 24 (Suppl 1):S1-S106, 2007 (Erratum in J Neurotrauma 25:276-278, 2008)

4. Brickley MR, Shepherd JP: The relationship between alcohol intoxication, injury severity and Glasgow Coma Score in assault patients. Injury 26:311-314, 1995

5. Farshchian N, Farshchian F, Rezaei M: Correlation between Glasgow coma scale and brain CT-scan findings in traumatic patients. J Inj Violence Res 4 (3 Suppl 1):Paper No. 44, 2012 (Abstract)

6. Galbraith S, Murray WR, Patel AR, Knill-Jones R: The relationship between alcohol and head injury and its effect on the conscious level. Br J Surg 63:128-130, 1976

7. Lange RT, Iverson GL, Brubacher JR, Franzen MD: Effect of blood alcohol level on Glasgow Coma Scale scores following traumatic brain injury. Brain Inj 24:919-927, 2010

8. Maas AI, Dearden M, Teasdale GM, Braakman R, Cohadon F, Iannotti F, et al: EBIC-guidelines for management of severe head injury in adults. Acta Neurochir (Wien) 139:286294, 1997

9. Maas AI, Hukkelhoven CW, Marshall LF, Steyerberg EW: Prediction of outcome in traumatic brain injury with computed tomographic characteristics: a comparison between the computed tomographic classification and combinations of computed tomographic predictors. Neurosurgery 57:11731182,2005

10. Parry-Jones BL, Vaughan FL, Miles Cox W: Traumatic brain injury and substance misuse: a systematic review of prevalence and outcomes research (1994-2004). Neuropsychol Rehabil 16:537-560, 2006

11. Rosenberg JB, Shiloh AL, Savel RH, Eisen LA: Non-invasive methods of estimating intracranial pressure. Neurocrit Care 15:599-608, 2011

12. Rutland-Brown W, Langlois JA, Thomas KE, Xi YL: Incidence of traumatic brain injury in the United States, 2003. J Head Trauma Rehabil 21:544-548, 2006

13. Shahin H, Gopinath SP, Robertson CS: Influence of alcohol on early Glasgow Coma Scale in head-injured patients. J Trauma 69:1176-1181, 2010

14. Skandsen T, Kvistad KA, Solheim O, Lydersen S, Strand IH, Vik A: Prognostic value of magnetic resonance imaging 
in moderate and severe head injury: a prospective study of early MRI findings and one-year outcome. J Neurotrauma 28:691-699, 2011

15. Skandsen T, Kvistad KA, Solheim O, Strand IH, Folvik M, Vik A: Prevalence and impact of diffuse axonal injury in patients with moderate and severe head injury: a cohort study of early magnetic resonance imaging findings and 1-year outcome. Clinical article. J Neurosurg 113:556-563, 2010

16. Skandsen T, Vik A: Subacute MR imaging: diffuse axonal injury, brain stem lesions and prognostic factors, in Sunderstrøm T, Grände PO, Juul N, et al (eds): Management of Severe Traumatic Brain Injury. Berlin: Springer-Verlag, 2012, pp 389-395

17. Sperry JL, Gentilello LM, Minei JP, Diaz-Arrastia RR, Friese RS, Shafi S: Waiting for the patient to "sober up": effect of alcohol intoxication on Glasgow Coma Scale score of brain injured patients. J Trauma 61:1305-1311, 2006

18. Stein SC, Spettell C: The Head Injury Severity Scale (HISS): a practical classification of closed-head injury. Brain Inj 9:437-444, 1995

19. Sternbach GL: The Glasgow coma scale. J Emerg Med 19:67-71, 2000

20. Stocchetti N, Pagan F, Calappi E, Canavesi K, Beretta L, Citerio G, et al: Inaccurate early assessment of neurological severity in head injury. J Neurotrauma 21:1131-1140, 2004

21. Stuke L, Diaz-Arrastia R, Gentilello LM, Shafi S: Effect of alcohol on Glasgow Coma Scale in head-injured patients. Ann Surg 245:651-655, 2007

22. Sullivan JB Jr, Hauptman M, Bronstein AC: Lack of observable intoxication in humans with high plasma alcohol concentrations. J Forensic Sci 32:1660-1665, 1987
23. Tagliaferri F, Compagnone C, Korsic M, Servadei F, Kraus J: A systematic review of brain injury epidemiology in Europe. Acta Neurochir (Wien) 148:255-268, 2006

24. Teasdale G, Jennett B: Assessment of coma and impaired consciousness. A practical scale. Lancet 2:81-84, 1974

25. Undén J, Ingebrigtsen T, Romner B: Scandinavian guidelines for initial management of minimal, mild and moderate head injuries in adults: an evidence and consensus-based update. BMC Med 11:50, 2013

\section{Author Contributions}

Author contributions to the study and manuscript preparation include the following. Conception and design: Moen, Rundhaug, Vik. Acquisition of data: Moen, Rundhaug, Skandsen, Lund, Hara, Vik. Analysis and interpretation of data: Moen, Rundhaug, Skandsen, Schirmer-Mikalsen, Lund, Vik. Drafting the article: Moen, Rundhaug, Skandsen, Schirmer-Mikalsen, Lund, Vik. Critically revising the article: all authors. Reviewed submitted version of manuscript: all authors. Statistical analysis: Moen, Rundhaug, Skandsen, Vik. Administrative/technical/material support: Rundhaug, Vik. Study supervision: Moen, Rundhaug, Skandsen, Vik.

\section{Correspondence}

Kent Gøran Moen, Department of Neuroscience, Norwegian University of Science and Technology, Postboks 3250 Sluppen, N-7491 Trondheim, Norway. email: kent.g.moen@ntnu.no. 